\title{
The Laplace transform solution
}

\section{of a one dimensional groundwater recharge by spreading}

\author{
A. P. Verma $(*)$
}

Ricevuto il 21 Gennaio 1969

Rinssunto. - Un'espressione analitica per la distribuzione del contenuto di unidita, in un problema di carico unidimensionale verticale di acqua sotterranea, ì stata ottenuta mediante il metodo di trasformazione di Laplace. Il coefliciente medio di diffusibilità, calcolato sull'intera gramna di valori di unidità contenuta si considera come costante, mentre si assume una variazione lineare di permeabilita con contenuto di umidita.

SUMMARY. - An analytical expression for the moisture content distribution, in a problem of one dimensional vertical groundwater recharge, has been obtained by using the Laplace transform method. The average diffusivity coefficient over the whole range of moisture content is regarded as constant, and a linear variation of permeability with moisture content is assumed.

\section{1. - INTRODUCTION.}

Recently Klute $\left({ }^{1,2}\right)$ and Sarma (3) have discussed the numerical methods of solution for the flow of water in partially saturated porous media. In the present paper we have obtained an analytical solution of a one dimensional problem of groundwater recharge by spreading.

We consider here that the recharge takes place over a large basin of such geological location that the sides are limited by rigid boundaries, and the bottom by a thick layer of watertable. Under these circumstances, water, from the spreading grounds, will flow vertically

(*) Department of Mathematies, Faculty of Technology and Engineering, M. s. University of Baroda, Baroda, India. 
downwards through the unsaturated porous media. It is assumed that the diffusivity coefficient is equivalent to its average value over the whole range of moisture content, and the permeability of the media is a continuous linear function of the moisture content.

The nonlinear partial differential equation for moisture content has been solved by employing the Japlace transform technique, and an analytical expression for moisture distribution is given.

2. - Formulation OF THE BOUNDARY VALUE PROBLEM.

Following Klute ( $\left.{ }^{1}\right)$, we may wite the fundamental equations as below.

The equation of continuity for an unsaturated medium is given by

$$
\frac{\partial}{\partial t}\left(\varrho_{s} \theta\right)=-\nabla \cdot M
$$

where $\varrho_{s}$ is the bulk density of the medium, 0 is its moisture content on a dry weight basis, and $W$ is the mass flux of moisture.

Darcy's law governing the motion of water in a porous medium may be written as:

$$
V=-K \nabla \phi
$$

where $v d$ represents the grradient of the total moisture potential, $V$ the volume flux of moisture, and $K$ the coefficient of aqueous conductivity.

From equations [2.1] and [2.2], we get:

$$
\frac{\partial}{\partial i}\left(\varrho_{s} \theta\right)=\nabla \cdot(\varrho K V \phi)
$$

where $n$ is the fluid density.

Since, in the present problem, flow takes place only in the vertical direction, therefore equation [2.3] reduces to:

$$
\varrho s \frac{\partial \theta}{\partial t}=\frac{\partial}{\partial z}\left(\varrho K \frac{\partial \psi}{\partial z}\right)-\frac{\partial}{\partial z}(\varrho K g),
$$

where $\psi$ is the pressure (capillary) potential, $g$ is the gravitation content, and $\phi=\dddot{r}-g Z$. The positive direction of $Z$ axis is the same as that of the gravity. 
Considering 0 and $\psi$ to be connected by a single valued function, we may write equation [4] as:

$$
\frac{\partial \theta}{\partial t}=\frac{\partial}{\partial z}\left(\dot{\partial} \frac{\partial \theta}{\partial z}\right)+\frac{\varrho}{\varrho_{s}} g \frac{\partial K}{\partial Z},
$$

where $I)=\frac{e}{\partial_{s}} K \frac{\partial \psi}{\partial \theta}$, and is called the diffusivity coefficient.

Replacing $D$ by its average value $D_{a}$, and assuming $K=k_{0} 0$, $K_{0}=232$ (as in [3] and [1]), we have:

$$
\frac{\partial \theta}{\partial t}=I_{a} \frac{\partial^{2} \theta}{\partial t^{2}}-\frac{\stackrel{\sim}{\varrho}}{\varrho_{s}} K_{o} \frac{\partial \theta}{\partial \widetilde{Z}} .
$$

Considering the watertable to be situated at a depth $L$, and putting:

$$
\frac{Z}{L}=\xi, \quad \frac{t D_{a}}{L^{2}}=T
$$

we may write the boundary value problem as:

$$
\begin{gathered}
\frac{\partial 0}{\partial T}-\frac{\partial^{2} \theta}{\partial \xi^{2}}-\frac{\varrho}{O_{s}} \frac{K_{0}}{D_{a}} \frac{\partial \theta}{\partial \xi}, \\
0(0, T)=0, \quad 0(1, T)=1, \\
O(\xi, 0)=0,
\end{gathered}
$$

where the moisture content thronghout the region is zero initially, at the layer $Z=0$ it is $0_{o}$, and at the water table $(Z=L)$ it is assumed to remain $100 \%$ throughout the process of investigation. It may be remarked that the effect of capillary action at the stationary groundwater level, being small, is neglected.

\section{3. - ANalytical solution.}

\section{Setting}

$$
\frac{\varrho}{\varrho_{s}} \frac{K_{0}}{D_{a}}=\beta
$$

in equation [:.7], we get:

$$
\frac{\partial \theta}{\partial T}=\frac{\partial^{2} \theta}{\partial \xi^{2}}-\beta \frac{\partial \theta}{\partial \xi},
$$


On multiplying each term of equation [3.1] by $e^{-S T} d T$, integrating the result from zero to infinity, and using condition [2.9], we obtain

$$
\frac{d^{2} \theta}{d \xi^{2}}-\hat{\rho}-\frac{d \theta}{d \xi}-S \theta=0
$$

where

$$
\theta(\xi, S)=\int_{0}^{\infty} e^{-s i} \theta(\xi, T) d T
$$

represents the Iaplace transform of $\theta(\xi, T)$.

The Laplace transformation of the boundary conditions [2.8] yields

$$
\theta(0, S)=\frac{0_{o}}{S} \quad, \quad \bar{\theta}(1, S)=\frac{1}{S} .
$$

Since equation [3.2] is a linear equation with constant coefficient, we may write its general solution as:

$\eta(\xi, S)=\left[E \cosh \left(\xi / \beta^{2} / 4+S\right)+F \sinh \left(\xi / / \beta^{2} / 4+S\right)\right] e^{\frac{\beta}{2} \xi}$,

where $E$ and $F$ are constants of integration. For evaluating $E$ and $F$, we apply conditions [3.3] to equation [3.4], so that, after some simplification, we have:

$$
E=\frac{\theta_{0}}{S}, \quad F=\frac{\frac{1}{S} e^{-\frac{\beta}{2}}-\frac{\theta_{o}}{S} \cosh \left(1 \beta^{2} / 4+S\right)}{\sinh \left(\sqrt{\beta^{2} / 4+S}\right)} .
$$

Substituting these values in equation [3.4], we have:

$$
\begin{aligned}
\bar{\theta}(\xi, S)=e^{\frac{\beta}{2} \xi}\left\{\frac{\theta_{0}}{S} \frac{\sinh (1-\xi) / \beta^{2} / 4+S}{\sinh \sqrt{\beta^{2} / 4+S}}\right\}+ \\
+e^{-(1-\xi)}\left\{\frac{\sinh \xi / \beta^{2} / 4+S}{S \sinh / \beta^{2} / 4+S}\right\}
\end{aligned}
$$


The inverse transform $\left(L^{-1}\right)$ of the right hand side terms in equation [3.5] may be determined by recalling a standard result [4] viz..

$$
L^{-1}\left|\frac{\zeta(S)}{\eta(S)}\right|=\sum_{n=0}^{\infty} \frac{\zeta\left(S_{n}\right)}{n^{1}\left(S_{n}\right)} e^{S_{n} T}
$$

where $\zeta(S)$ and $\eta(S)$ represents two entire transcendental functions such that degree of $\eta(S)$ is atleast one greater in $S$ (when expressed as power series) than that of $\zeta(S), S_{n}$ is a simple pole of $\frac{\zeta\left(S^{\prime}\right)}{\eta(5)}$, and $\eta^{1}\left(S_{n}\right)$ denotes the value of $\frac{d \eta(S)}{d S}$ at $S=S_{n}$. Putting:

$$
\frac{\zeta(S)}{\eta(S)}=\frac{\sinh \left(\xi \sqrt{\beta^{2} / 4+S}\right)}{S \sinh \left(\sqrt{\beta^{2} / 4+S}\right)}=\frac{\sinh \left(i \xi 1 / \beta^{2} / 4+S\right)}{S \sinh \left(i \sqrt{\beta^{2} / 4+S}\right)},
$$

and noting that the roots of equation

$$
\sinh \left(\gamma^{\prime} \beta^{2} / 4+S\right)=0
$$

are given by

$$
S_{n}=-\beta^{2} / 4-n^{2} \pi^{2}
$$

we may write:

$$
\left\{\begin{array}{c}
\zeta\left(S_{n}\right)=\sin (n \pi \xi), \quad \zeta(0)=i \sinh \frac{p}{2} \xi \\
\eta^{1}\left(S_{n}\right)=\frac{(-1)^{n}\left(\beta^{2} / 4+n^{2} \pi^{2}\right)}{2 \pi i \pi}, \eta^{1}(0)=i \sinh (\beta / 2) .
\end{array}\right.
$$

From equations [3.6], [3.7] and [3.8], we get:

$$
\begin{gathered}
L^{-1}\left|\frac{\sinh \xi \sqrt{\beta^{2} / 4+S}}{S \sinh \sqrt{\beta^{2} / 4+S}}\right|=\frac{\sinh \frac{\beta}{2}}{\sinh \beta / 2}+ \\
+2 \pi \sum_{n=1}^{\infty} \frac{(-1)^{n} n \sin (n \pi \xi)}{\beta^{2} / 4+n^{2} \pi^{2}}=-\left(\beta^{2} / 4+n^{2} \pi^{2}\right) T .
\end{gathered}
$$


Similarly, we have:

$$
\begin{aligned}
& L_{-1}\left|\frac{\sinh (1-\xi) / \overline{\beta^{2} / 4+S}}{s \sinh 1 \beta^{2} / 4+S}=\frac{\sinh \frac{\beta}{2}(1-\xi)}{\sinh \beta / 2}\right|- \\
& -2 \pi \sum_{n=1}^{\infty} \frac{n \sinh (n \pi \xi)}{\beta^{2} / 4+n^{2} \pi^{2}}{ }^{-}\left(\beta^{2} / 4+n^{2} \pi^{2}\right) T .
\end{aligned}
$$

The inverse transformation of equation [3.5] with the help of equation [3.9] and [3.10], yields:

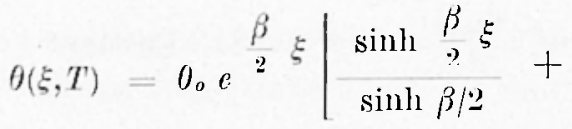

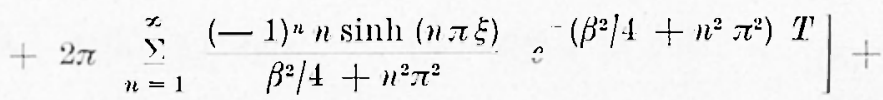

$$
\begin{aligned}
& +e^{-\frac{\beta}{2}(1-\xi) \mid \frac{\sinh \frac{\beta}{2}(1-\xi)}{\sinh \beta / 2}-} \\
& \left.-9 \pi \sum_{n=1}^{\infty} \frac{n \sin (n \pi \xi)}{\beta^{2} / 4+n^{2} \pi^{2}} e^{-\left(\beta^{2} / 4+n^{2} \pi^{2}\right) T}\right] .
\end{aligned}
$$

This is the desired analytical expression for the moisture content distribution.

It follows immediately from equation [3.11] that the graph of the moisture content versus distance (for griven values of time, say $t=1,4,16 \ldots$ etc.) may be easily drawn (as in [1]). A numerical illustration is equally obvious. However, these are not included here due to our particular interest in only an analytical solution.

\section{4. - Conchusion.}

An analytical solution for the nonlinear differential equation governing moisture content distribution las been obtained, by using Laplace transform method, for those cases of gromndwater recharge by spreading where the flow is essentially one dimensional, and in the vertical direction.

Though no numerical illustration is included in the present paper (because of our particular interest) yet the convenient form of the moisture content expression is immediately evident. 


\section{REFERENCES}

(1) KuUte A., A numerical method for solving the flow equation for water in unsaturated materials. "Soil seience ,. 73. 2, 105. (1952).

${ }^{2}$ KLote A., Some theoretical aspects of the flow of nater in unsaluraled soils. "Proc. Soil science Society", 16, 2, 144, (1952).

(3) SARMa S. V. K.. Problem of partially saturated unsteady state of flow through porous media with special rejerence to grounduater recharge by spreading. "J. sei. Engg. Research", IX, 1, 69, (1965).

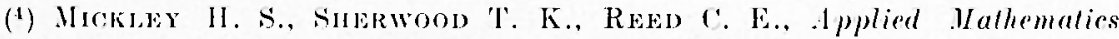
in chemical Engineering. M. Graw-Hill, 296, New York, 1957.

\section{NOMENCLATURT}

$D=$ diffusivity coefficient $\left(\mathrm{cm}^{2} \mathrm{sec}^{-1}\right)$

$D_{a}=$ average value of the diflusivity coefficient over the whole lange of moisture content (cm $\left.\mathrm{cm}^{2} \mathrm{ser}^{-1}\right)$

$K_{0}=$ slope of the permeability os moisture content plot (cm sec-1)

$K=$ permeability coefficient (cm sec-1)

$L=$ depth of permeable stratum (cm)

$M=$ mass flux of moisture (gm)

$t=$ time (sec)

$T^{\prime}=$ time (dimensionless)

$V^{\prime}=$ velocity of flow of water (cm sec $\left.{ }^{-1}\right)$

$Z \quad=$ depth of penetration of water at any instant $t(\mathrm{~cm})$

$\beta=$ a flow parameter $\left(\mathrm{cm}^{2}\right)$

$\xi \quad=$ penetration depth (dimensionless)

$\phi=$ total pressure potential $\left(\mathrm{cm} \mathrm{sec}^{-2}\right)$

$\psi=$ arpillary potential (cm sec $\left.{ }^{-2}\right)$

$g=$ acceleration due to gravity $\left(\mathrm{cm}\right.$ sec $\left.{ }^{-2}\right)$

D = mass density of water (gm)

$\varrho_{\delta} \quad=$ bulk density of the medium on dry weight basis $\left(\mathrm{gm} \mathrm{cm}^{-3}\right)$

$0_{0}=$ moisture content at $Z=0$ for all time $(\mathrm{gm} / \mathrm{gm})$

$0=$ moisture content at any depth $Z\left(g m \operatorname{mm}^{-1}\right)$

$V=$ vector operator $=\vec{\imath} \frac{\partial}{\partial x}+\vec{\jmath} \frac{\partial}{\partial y}+\vec{K} \frac{\lambda}{\partial z} \overrightarrow{i j}, \vec{j}, \vec{K}$ are unit vectors) 